\title{
Vitamin D Deficiency Is Associated with Endoscopic Severity in Patients with Crohn's Disease
}

\author{
Lingna Ye, ${ }^{1,2}$ Ziwen Lin, $^{2}$ Jing Liu, ${ }^{2}$ and Qian $\mathrm{Cao}^{2}$ \\ ${ }^{1}$ Department of Gastroenterology, Xiasha Branch of Sir Run Run Shaw Hospital, School of Medicine, Zhejiang University, \\ Hangzhou, China \\ ${ }^{2}$ Inflammatory Bowel Disease Center, Sir Run Run Shaw Hospital, School of Medicine, Zhejiang University, Hangzhou, China
}

Correspondence should be addressed to Qian Cao; 0000232@zju.edu.cn

Received 18 July 2017; Revised 25 September 2017; Accepted 8 October 2017; Published 16 November 2017

Academic Editor: Francesco Selvaggi

Copyright (c) 2017 Lingna Ye et al. This is an open access article distributed under the Creative Commons Attribution License, which permits unrestricted use, distribution, and reproduction in any medium, provided the original work is properly cited.

Background and Aims. Vitamin D deficiency is common in patients with Crohn's disease and is associated with disease activity. Relationship between vitamin D and endoscopic disease activity is unknown. The aim of the study is to determine the association between vitamin D status and endoscopic disease activity in CD patients. Methods. Consecutive hospitalized CD patients from 2014 to 2016 who received vitamin D assessment and colonoscopy were retrospectively evaluated. Clinical disease activity was assessed by Crohn's disease activity index and C-reactive protein. Endoscopic activity was calculated using simple endoscopic score for Crohn's disease. Results. Median serum 25OHD level of 131 patients was lower than healthy controls [21.1 nmol/L (11.8-32.3) versus $49.9 \mathrm{nmol} / \mathrm{L}(44.9-57.4), P=0.007] .125$ (95\%) patients had vitamin D deficiency and the rest (5\%) had vitamin D insufficiency. Serum 25OHD was inversely correlated with CRP $(r=-0.308, P<0.001)$, CDAI $(r=-0.582$, $P<0.001)$, SES-CD $(r=-0.294, P=0.001)$, and endoscopic severity stratified by SES-CD $(P=0.001)$. Conclusion. Vitamin D deficiency was prevalent among hospitalized CD patients. Vitamin D levels were inversely correlated with endoscopic disease activity. Vitamin D status could be a biomarker in assessing disease activity among hospitalized CD patients in addition to CDAI and CRP.

\section{Introduction}

Vitamin D (25OHD), as a potential immune modulator, is a risk factor for Crohn's disease (CD). Vitamin D influences both $\mathrm{CD}$ onset [1] and progression [2] of disease activity [3]. However, evidence regarding associations between vitamin $\mathrm{D}$ status and disease activity in $\mathrm{CD}$ has been inconsistent. A recent meta-analysis [4] showed CD patients have lower levels of vitamin D compared to healthy controls, but such difference disappeared when compared to nonhealthy controls [5-7]. Furthermore, vitamin D levels may correlate with disease activity among $\mathrm{CD}$ patients $[8,9]$.

Historically, clinical indices (Crohn's disease activity index (CDAI), Harvey-Bradshaw index (HBI)) and systemic inflammatory markers (C-reactive protein (CRP)) have been used to assess disease activity in $\mathrm{CD}$ and have been found to correlate with levels of vitamin D $[10,11]$. However, compared to conventional clinical index and CRP in CD, endoscopic assessment of disease activity has been thought to be superior in discriminating disease severity and degrees of mucosal inflammation in clinical practice. For example, mucosal healing under endoscopy examination is strongly associated with favorable disease outcome and therefore it has become an increasingly important therapeutic goal for CD patients. Simple endoscopic score for Crohn's disease (SES-CD) is developed to assess mucosal inflammation in CD. SES-CD demonstrated high level of agreement among different endoscopists [12]. Furthermore, improvement of SES-CD scores in therapeutic trials was associated with corticosteroid-free clinical remission in patients with CD [13].

Vitamin D levels as an immune modulator may predict mucosal activity in patients in CD. To our knowledge, association between 25OHD and endoscopic disease severity 
TABle 1: Definitions of simple endoscopic score for Crohn's disease.

\begin{tabular}{lcccc}
\hline & \multicolumn{4}{c}{ Simple endoscopic score for Crohn's disease values } \\
\hline Size of ulcers & 0 & 1 & 2 & 3 \\
Ulcerated surface & None & Aphthous ulcers $(0.1-0.5 \mathrm{~cm})$ & Large ulcers $(0.5-2 \mathrm{~cm})$ & Very large ulcers $(>2 \mathrm{~cm})$ \\
Affected surface & None & $<10 \%$ & $10-30 \%$ & $>30 \%$ \\
Presence of narrowings & Unaffected segment & $<50 \%$ & $50-75 \%$ & $>75 \%$ \\
\hline
\end{tabular}

assessed by SES-CD is unknown. Therefore, the primary aim of the current study is to investigate the association between vitamin $\mathrm{D}$ levels and endoscopic disease activity in CD using SES-CD. The secondary aim is to evaluate the association between vitamin D levels and other disease activity assessment tool such as CDAI, CRP, and albumin.

\section{Material and Methods}

2.1. Patient Population. All participants provided consents to participate in the current study. This study is approved by the ethics committee of our hospital. Consecutive CD patients hospitalized between March 2014 and July 2016 at the inflammatory bowel disease inpatient center of our hospital who received vitamin $\mathrm{D}$ level evaluation and colonoscopy were retrospectively analyzed. The $25 \mathrm{OHD}$ levels were compared in CD patients to 40 healthy controls and are comparable in terms of age, sex, and timing of the test. Inclusion criteria included (1) diagnosed as CD based on a careful evaluation on patients' history, imaging, lab tests, and endoscopic and pathology results, (2) hospitalized patients between March 2014 and July 2016 admitted to the inflammatory bowel disease inpatient center, (3) age $\geq 16$ years, (4) those patients with colonic (L2) or ileocolonic (L3) disease according to Montreal classification. Patients who had previous extensive small intestinal surgery or short bowel syndrome were excluded given that vitamin D deficiency may be attributed to malabsorption. Clinical and endoscopic data were obtained to characterize the patient's clinical course.

2.2. Definitions of Crohn's Disease Activity. Crohn's disease activity was evaluated by a combination of clinical, biochemical, and endoscopic assessment. Crohn's disease activity index (CDAI) was calculated for each patient; disease severity was stratified to four categories according to CDAI scores, with $\mathrm{CDAI}<150$ classified as remission, CDAI between 150 and 220 as mildly active, CDAI between 220 and 450 as moderately active, and CDAI above 450 as severely active [14]. C-reactive protein (CRP) levels were obtained in all patients at presentation. Abnormal CRP was defined as a level of above $5 \mathrm{mg} / \mathrm{L}$. Albumin is considered as a supplementary index for disease activity, and $<35 \mathrm{~g} / \mathrm{L}$ was considered abnormal. Colonoscopy was performed by one gastroenterologist, and endoscopic scores were graded by another experienced gastroenterologist specializing in IBD who reviewed all relevant stored images.

Disease activity assessed by endoscopy was calculated using the simple endoscopic score for Crohn's disease (SES-CD) (Table 1) [15]. Each of the four SES-CD variables (size of ulcers, ulcerated surface, affected surface, and the presence of narrowing) is scored from 0 to 3 for each of the five anatomical segments (terminal ileum, right colon, transverse colon, left colon, and rectum). The total SES-CD score is the sum of scores of each variable from the five locations, raging from 0 to 56 , and is further categorized as no active disease (0-2), mildly active disease [3-6], moderately active disease [7-15], and severely active disease (>15) [15].

2.3. Definitions of Vitamin D Deficiency or Insufficiency. Serum 25OHD concentration was measured with radioimmunoassay and automeasured by using the Food and Drug Administration-approved Roche cobas 8000 automatic biochemical analyzer in the clinical laboratory of our hospital. According to the endocrine society clinical practice guideline [16], vitamin $\mathrm{D}$ deficiency is defined as a serum level of $25 \mathrm{OHD}$ lower than $50 \mathrm{nmol} / \mathrm{L}$, and a serum level above $50 \mathrm{nmol} / \mathrm{L}$ but lower than $75 \mathrm{nmol} / \mathrm{L}$ is classified as vitamin D insufficiency.

2.4. Statistics. Continuous variables following normal or nonnormal distributions were interpreted as mean with standard deviations, and median with quartiles, respectively. Categorical variables were presented as proportions. Kruskal-Wallis test was used to compare nonparametric variables between groups. Mann-Whitney $U$ test was used to compare bivariate variables. Kruskal-Wallis test and Spearman's rho were used to compare across categories of independent samples. Statistical significance was considered as a $P$ value of lower than 0.05. Statistical analysis was performed with SPSS (version 22.0, Chicago, IL).

\section{Results}

3.1. Characteristics of Patients and Healthy Controls. A total of 131 patients with CD met the inclusion criteria. Forty healthy controls were subsequently recruited. The two groups were not statistically different in age, sex, timing of the assay, nonsmoking, and BMI (Table 2). Two patients were excluded due to history of extensive small bowel disease surgery. The characteristics of 131 patients are shown in Table 3. The median age was 27, 96 patients (73\%) were males, and 120 (91.6\%) were nonsmokers. The median time of disease duration was 2 years, and 105 patients (80.2\%) were newly diagnosed with CD. Eight patients (6.1\%) had colonic disease and 123 (93.9\%) had ileocolonic disease. Of the 26 patients who was diagnosed before March 2014, 6 (23.1\%) were being treated with corticosteroids, 12 (46.2\%) 
TABLE 2: Comparisons of characteristics between patient group and healthy controls.

\begin{tabular}{|c|c|c|c|}
\hline Characteristics & $\begin{array}{l}\text { Healthy controls } \\
N=40(\%) \text { patients with colorectal cancer }\end{array}$ & Patients with Crohn's disease $N=131(\%)$ & $P$ value \\
\hline Age [median (quartiles)] & $27(18-57)$ & $27(22-35)$ & 0.646 \\
\hline Male & $27(67.5 \%)$ & $96(73 \%)$ & 0.575 \\
\hline $\begin{array}{l}\text { Serum } 25 \mathrm{OHD} \text { level, nmol/L } \\
\text { [median (quartiles)] }\end{array}$ & $49.9(44.9-57.4)$ & $21.1(11.8-32.3)$ & 0.007 \\
\hline $\begin{array}{l}\text { Detected in spring and winter/ } \\
\text { Detected in summer and autumn }\end{array}$ & $17 / 23$ & $64 / 67$ & 0.588 \\
\hline Nonsmoking $[n(\%)]$ & $32(80 \%)$ & $120(91.6 \%)$ & 0.302 \\
\hline BMI [median (quartiles)] & $20(17.3-22.1)$ & $19.0(17.0-21.3)$ & 0.701 \\
\hline
\end{tabular}

BMI: body mass index.

TABLE 3: Clinical characteristics of patient group.

\begin{tabular}{lc}
\hline Characteristics & $N=131(\%)$ \\
\hline Years diagnosed [median (quartiles)] & $2(1-5)$ \\
Montreal classification $[n(\%)]$ & \\
Age at diagnosis & $9(6.8)$ \\
A1 (<17 years) & $99(75.6)$ \\
A2 (17-40 years) & $23(17.6)$ \\
A3 ( $\geq 40$ years) & \\
Disease location $[n(\%)]$ & $8(6.1)$ \\
L2 (colonic) & $123(93.9)$ \\
L3 (ileocolonic) & \\
Disease behavior [n (\%)] & $97(74)$ \\
B1 (nonstricturing, nonpenetrating) & $24(18.3)$ \\
B2 (stricturing) & $4(3.1)$ \\
B3 (penetrating) & $6(4.6)$ \\
B2 + B3(stricturing and penetrating) & \\
Disease activity & $241(84)$ \\
CDAI score [mean (SD)] & $16.6(6.4-37.1)$ \\
CRP, mg/L [median (quartiles)] & $34.8(6.2)$ \\
Albumin, g/L [mean (SD)] & $11(6-17)$ \\
SES-CD [median (quartiles)] & $21.1(11.8-32.3)$ \\
Serum 25OHD [nmol/L, median (quartiles)] &
\end{tabular}

SES-CD: the simple endoscopic score for Crohn's disease, CDAI: Crohn's disease activity index, and CRP: C-reactive protein.

received immunomodulators, 5 (19.2\%) received mesalazine, and $3(11.5 \%)$ received biologics at presentation.

3.2. Prevalence of Vitamin D Deficiency. The median serum $25 \mathrm{OHD}$ level of 131 patients was lower than healthy controls [21.1 nmol/L (11.8-32.3) versus $49.9 \mathrm{nmol} / \mathrm{L}$ (44.9-57.4), $P=0.007]$. All patients had suboptimal levels of vitamin D including 125 (95\%) with vitamin D deficiency and remaining 6 (5\%) with vitamin D insufficiency.

3.3. Vitamin D Status and Disease Severity Measured by SES-CD. Endoscopic evaluation of disease severity showed that $8.4 \%$ of the patients was in clinical remission, $17.6 \%$ were mildly active, $43.5 \%$ had moderately active disease,
TABLE 4: Association between vitamin $\mathrm{D}$ status and disease severity in Crohn's disease, measured by CRP, CDAI, albumin, and SES-CD.

\begin{tabular}{lcc}
\hline & Spearman's rho & $P$ value \\
\hline 25OHD and SES-CD & -0.294 & 0.001 \\
25OHD and CDAI & -0.582 & $<0.001$ \\
25OHD and CRP & -0.308 & $<0.001$ \\
25OHD and albumin & -0.484 & $<0.001$ \\
\hline SES-CD: the simple endoscopic score for Crohn's disease, CDAI: Crohn's \\
disease activity index, and CRP: C-reactive protein.
\end{tabular}

and $30.5 \%$ had severely active disease. Disease severity, evaluated by SES-CD, was inversely correlated with 25OHD levels $(r=-0.294, P=0.001)$ (Table 4$)$.

Serum levels of $25 \mathrm{OHD}$ were lower in patients with active disease compared to patients in remission. For patients in remission, mild active disease, moderate active disease, and severe active disease, the serum 25OHD levels were $23.7 \mathrm{nmol} / \mathrm{L}, 32.3 \mathrm{nmol} / \mathrm{L}, 21.4 \mathrm{nmol} / \mathrm{L}$, and $17.5 \mathrm{nmol} / \mathrm{L}$, respectively $(P=0.001)$ (Figure 1$)$.

3.4. Vitamin D Status and Disease Severity Measured by CRP, CDAI, and Albumin. The majority of subjects (82.4\%) had a CRP level of higher than 5, indicating active inflammation, and the remaining $17.6 \%$ was in remission, indicated as a CRP level of lower than 5. At presentation, $115(88 \%)$ patients were experiencing flare and $16(12 \%)$ were in clinical remission, as indicated by CDAI. Of those with active disease, $32 \%$ was mildly active, $53.4 \%$ had moderately active disease, and $2.2 \%$ had severely active disease.

Disease severity, evaluated by CRP, CDAI, and albumin, was inversely correlated with 25OHD levels (Table 4). For patients in clinical remission as defined by a CDAI score of lower than 150, the median serum level of $25 \mathrm{OHD}$ was $33.1 \mathrm{nmol} / \mathrm{L}$, whereas in patients with mild disease and moderately active disease, the levels were lower $(29.8 \mathrm{nmol} / \mathrm{L}$ and $14.8 \mathrm{nmol} / \mathrm{L}$, respectively, $P<0.001$ ) (Figure 2 ).

\section{Discussion}

Lower serum 25OHD concentration may be associated with greater disease activity $[2,17]$, poorer disease course, and worse outcomes in CD patients [18]. Many researches had 


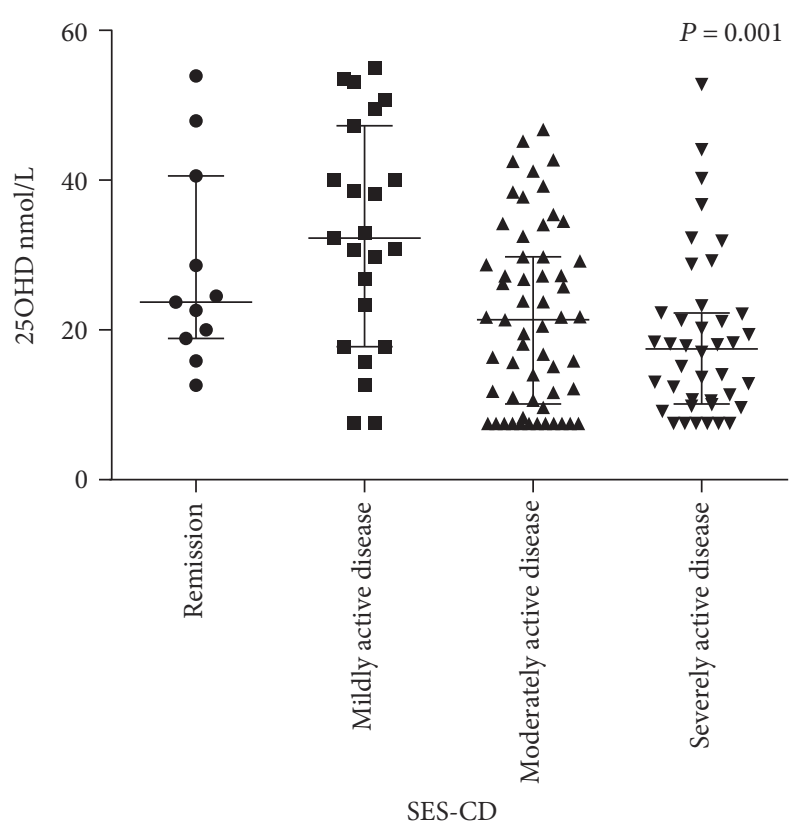

FIGURE 1: Comparison of serum 25OHD level of patients among clinical remission group, mildly active group, moderately active group, and severely active group stratified by SES-CD scores, $P=0.001$.

been carried out to explore the relationships between vitamin $\mathrm{D}$ status, disease activity, and systemic markers of inflammation $[2,10,17,19,20]$, and the results were inconsistent [3, 21]. Most studies have reported an inverse correlation between vitamin $\mathrm{D}$ status and disease activity in terms of HBI scores [11, 17, 19], CDAI, and CRP [17]. Yet, some other studies failed to demonstrate such associations between 25OHD with either CRP or CDAI [21-23]. Our study shows that disease severity, evaluated by systematic inflammation CRP, CDAI, and albumin, was strongly and inversely correlated with 25OHD levels, which is consistent with most studies.

To further investigate the association between vitamin $\mathrm{D}$ levels and clinical disease severity, we adopted SES-CD, in addition to CDAI and CRP levels; SES-CD was chosen to assess disease activity and mucosal inflammation. SES-CD is superior to CRP and CDAI in identifying various degrees of mucosal inflammation as it provides direct visualization of intestine mucosa. To our knowledge, there are no data that elucidate the relationship between vitamin $\mathrm{D}$ levels and endoscopic inflammation in patients with Crohn's disease. Our study is the first to report an inverse correlation between SES-CD and serum 25OHD concentrations, after analyzing the data from 131 patients with CD. Moreover, we found a significant, inverse association between serum 25OHD and four SES-CD categories, which indicated various degrees of disease severity.

Our study also finds that vitamin D deficiency is present in as many as $95.4 \%$ of inpatients with CD. A recent metaanalysis reported that the prevalence of vitamin $\mathrm{D}$ deficiency was $57.7 \%$ in CD patients [4]. A recent study shows that vitamin D deficiency accounts for 53\% (122/230) in outpatients with CD [24]. The prevalence of vitamin D deficiency

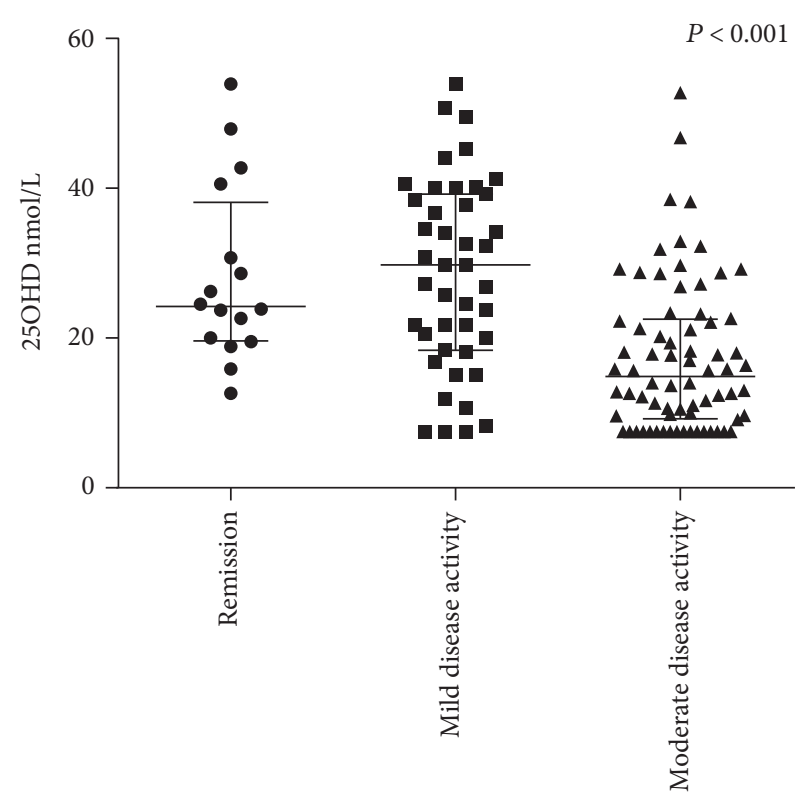

CDAI

FIgURE 2: Comparison of serum 25OHD levels among clinical remission group, mildly active group, and moderately active group stratified by CDAI scores, $P<0.001$.

in our study was higher, probably because participants in our study were all inpatient and had more severe disease and because their disease activity has not been controlled since most of the participants were newly diagnosed.

The current study has its limitation. The sample size is not large enough due to its retrospective analysis in nature. The controversial role of vitamin D in the assessment of disease activity as well as response to treatment still needs evidence from further well-designed prospective study.

In conclusion, among hospitalized patients with CD who received a colonoscopy, almost all the patients had vitamin $\mathrm{D}$ deficiency. In addition to CDAI and CRP levels, vitamin D levels are highly correlated with endoscopic disease activity assessed by simple endoscopic score for Crohn's disease. Vitamin D status may be a useful biomarker in assessing disease activity among hospitalized patients with Crohn's disease in clinical practice.

\section{Conflicts of Interest}

The authors declare that they have no conflicts of interest.

\section{Acknowledgments}

This is funded by the Health and Family Planning Commission of Hangzhou, Item no. 2015A52.

\section{References}

[1] A. N. Ananthakrishnan, H. Khalili, L. M. Higuchi et al., "Higher predicted vitamin D status is associated with reduced risk of Crohn's disease," Gastroenterology, vol. 142, no. 3, pp. 482-489, 2012. 
[2] S. P. Jørgensen, J. Agnholt, H. Glerup et al., "Clinical trial: vitamin D3 treatment in Crohn's disease - a randomized double-blind placebo-controlled study," Alimentary Pharmacology and Therapeutics, vol. 32, no. 3, pp. 377-383, 2010.

[3] M. Garg, O. Rosella, J. S. Lubel, and P. R. Gibson, "Association of circulating vitamin $\mathrm{D}$ concentrations with intestinal but not systemic inflammation in inflammatory bowel disease," Inflammatory Bowel Diseases, vol. 19, no. 12, pp. 2634-2643, 2013.

[4] M. Sadeghian, P. Saneei, F. Siassi, and A. Esmaillzadeh, "Vitamin D status in relation to Crohn's disease: metaanalysis of observational studies," Nutrition, vol. 32, no. 5, pp. 505-514, 2016.

[5] A. D. Harries, R. Brown, R. V. Heatley, L. A. Williams, S. Woodhead, and J. Rhodes, "Vitamin D status in Crohn's disease: association with nutrition and disease activity," Gut, vol. 26, no. 11, pp. 1197-1203, 1985.

[6] M. Tajika, A. Matsuura, T. Nakamura et al., "Risk factors for vitamin D deficiency in patients with Crohn's disease," Journal of Gastroenterology, vol. 39, no. 6, pp. 527-533, 2004.

[7] W. El-Matary, S. Sikora, and D. Spady, "Bone mineral density, vitamin $\mathrm{D}$, and disease activity in children newly diagnosed with inflammatory bowel disease," Digestive Diseases and Sciences, vol. 56, no. 3, pp. 825-829, 2011.

[8] Y. T. Fu, N. Chatur, C. Cheong-Lee, and B. Salh, "Hypovitaminosis $\mathrm{D}$ in adults with inflammatory bowel disease: potential role of ethnicity," Digestive Diseases and Sciences, vol. 57, no. 8, pp. 2144-2148, 2012.

[9] A. D. Levin, V. Wadhera, S. T. Leach et al., "Vitamin D deficiency in children with inflammatory bowel disease," Digestive Diseases and Sciences, vol. 56, no. 3, pp. 830-836, 2011.

[10] S. P. Jorgensen, C. L. Hvas, J. Agnholt, L. A. Christensen, L. Heickendorff, and J. F. Dahlerup, "Active Crohn's disease is associated with low vitamin D levels," Journal of Crohn's and Colitis, vol. 7, no. 10, pp. e407-e413, 2013.

[11] A. Raffner Basson, R. Swart, E. Jordaan, M. Mazinu, and G. Watermeyer, "Vitamin D deficiency increases the risk for moderate to severe disease activity in Crohn's disease patients in South Africa, measured by the Harvey Bradshaw index," Journal of the American College of Nutrition, vol. 35, no. 2, pp. 163-174, 2016.

[12] M. Daperno, G. D'haens, G. Van Assche et al., "Development and validation of a new, simplified endoscopic activity score for Crohn's disease: the SES-CD," Gastrointestinal Endoscopy, vol. 60, no. 4, pp. 505-512, 2004.

[13] M. Ferrante, J. F. Colombel, W. J. Sandborn et al., "Validation of endoscopic activity scores in patients with Crohn's disease based on a post hoc analysis of data from SONIC," Gastroenterology, vol. 145, no. 5, pp. 978-986.e5, 2013.

[14] W. R. Best, J. M. Becktel, J. W. Singleton, and F. Kern Jr., "Development of a Crohn's disease activity index. National cooperative Crohn's disease study," Gastroenterology, vol. 70, no. 3, pp. 439-444, 1976.

[15] D. N. Moskovitz, M. Daperno, V. Assche et al., "Defining and validating cut-offs for the simple endoscopic score for Crohn's disease," Gastroenterology, vol. 132, p. s1097, 2007.

[16] M. F. Holick, N. C. Binkley, H. A. Bischoff-Ferrari et al., "Evaluation, treatment, and prevention of vitamin D deficiency: an endocrine society clinical practice guideline," The Journal of
Clinical Endocrinology \& Metabolism, vol. 96, no. 7, pp. 1911-1930, 2011.

[17] M. Ham, M. S. Longhi, C. Lahiff, A. Cheifetz, S. Robson, and A. C. Moss, "Vitamin D levels in adults with Crohn's disease are responsive to disease activity and treatment," Inflammatory Bowel Diseases, vol. 20, no. 5, pp. 856-860, 2014.

[18] A. N. Ananthakrishnan, S. C. Cheng, T. Cai et al., "Association between reduced plasma 25 -hydroxy vitamin $\mathrm{D}$ and increased risk of cancer in patients with inflammatory bowel diseases," Clinical Gastroenterology and Hepatology, vol. 12, no. 5, pp. 821-827, 2014.

[19] A. Ulitsky, A. N. Ananthakrishnan, A. Naik et al., "Vitamin D deficiency in patients with inflammatory bowel disease: association with disease activity and quality of life," Journal of Parenteral and Enteral Nutrition, vol. 35, no. 3, pp. 308-316, 2011.

[20] A. J. Joseph, B. George, A. B. Pulimood, M. S. Seshadri, and A. Chacko, "25 (OH) vitamin D level in Crohn's disease: association with sun exposure \& disease activity," The Indian Journal of Medical Research, vol. 130, no. 2, pp. 133-137, 2009.

[21] P. Kelly, T. N. Suibhne, C. O'Morain, and M. O'Sullivan, "Vitamin D status and cytokine levels in patients with Crohn's disease," International Journal for Vitamin and Nutrition Research, vol. 81, no. 4, pp. 205-210, 2011.

[22] T. N. Suibhne, G. Cox, M. Healy, C. O'Morain, and M. O'Sullivan, "Vitamin D deficiency in Crohn's disease: prevalence, risk factors and supplement use in an outpatient setting," Journal of Crohn's and Colitis, vol. 6, no. 2, pp. 182-188, 2012.

[23] T. Raftery, M. Merrick, M. Healy et al., "Vitamin D status is associated with intestinal inflammation as measured by fecal calprotectin in Crohn's disease in clinical remission," Digestive Diseases and Sciences, vol. 60, no. 8, pp. 2427-2435, 2015.

[24] S. O. Frigstad, M. Høivik, J. Jahnsen et al., "Vitamin D deficiency in inflammatory bowel disease: prevalence and predictors in a Norwegian outpatient population," Scandinavian Journal of Gastroenterology, vol. 23, pp. 1-7, 2016. 


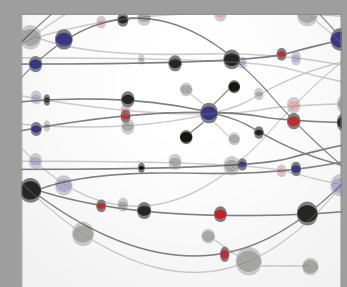

The Scientific World Journal
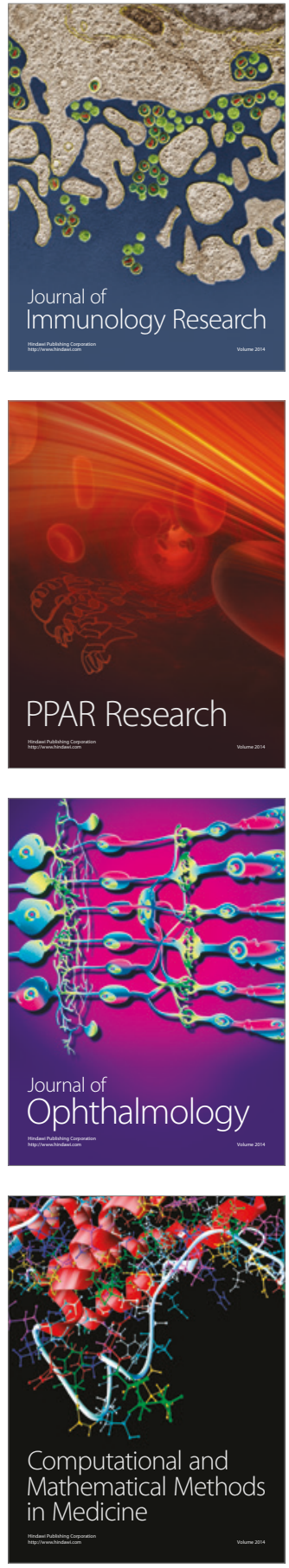

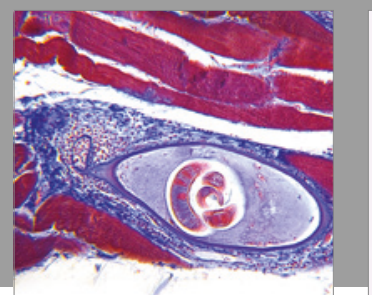

Gastroenterology Research and Practice
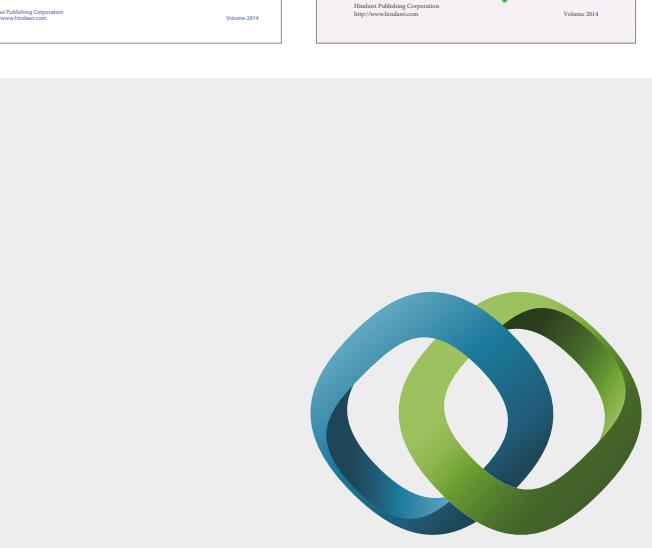

\section{Hindawi}

Submit your manuscripts at

https://www.hindawi.com
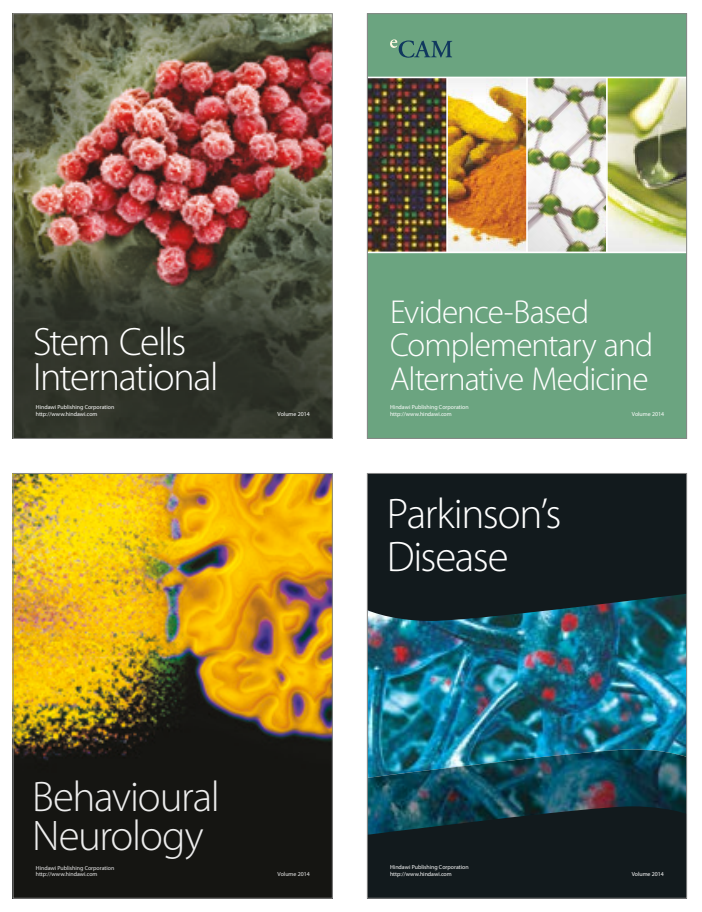
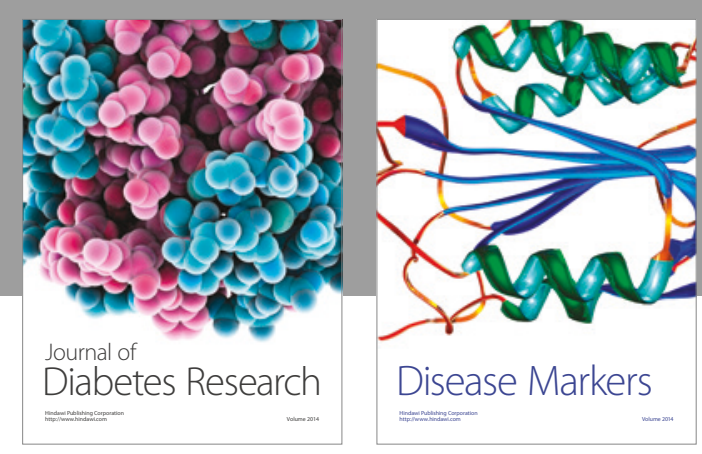

Disease Markers
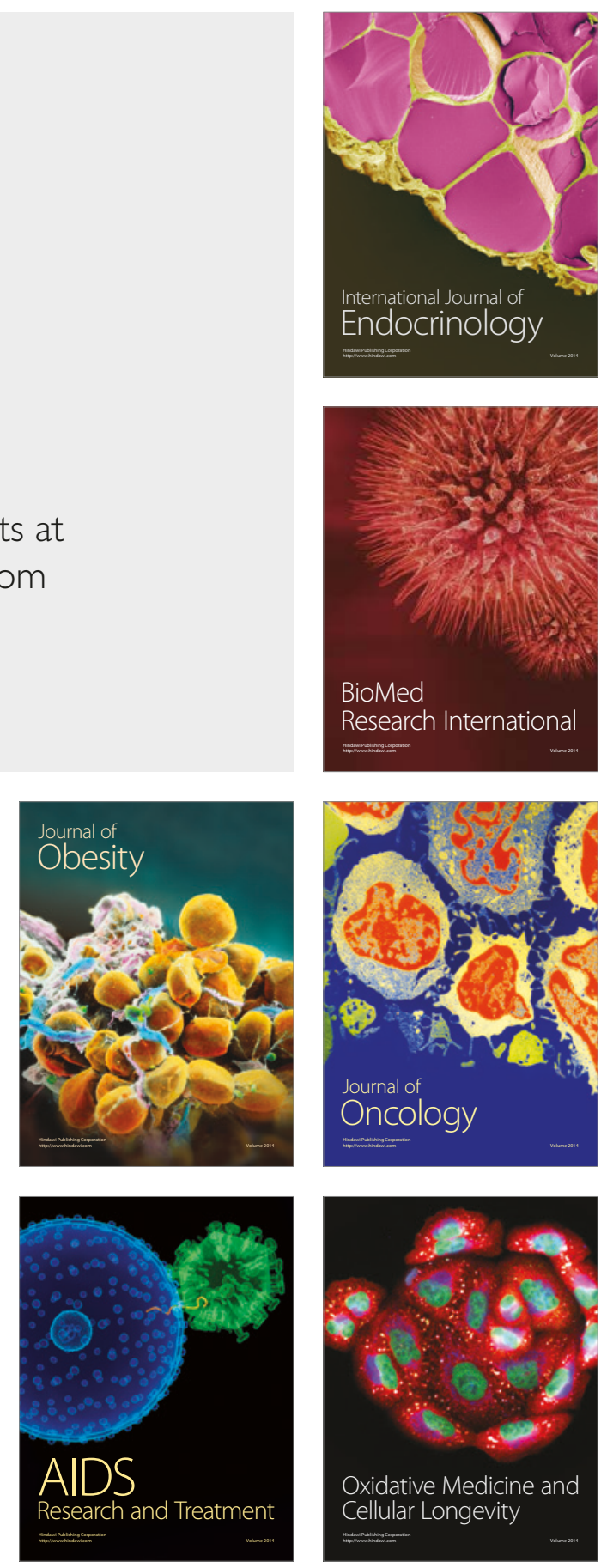